\title{
TRAVELLING-WAVE ANALYSIS OF A MODEL DESCRIBING TISSUE DEGRADATION BY BACTERIA
}

\author{
DANIELLE HILHORST, JOHN R. KING, AND MATTHIAS RÖGER
}

\begin{abstract}
We study travelling-wave solutions for a reaction-diffusion system arising as a model for host-tissue degradation by bacteria. This system consists of a parabolic equation coupled with an ordinary differential equation. For large values of the 'degradation-rate parameter' solutions are well approximated by solutions of a Stefan-like free boundary problem, for which travelling-wave solutions can be found explicitly. Our aim is to prove the existence of travelling waves for all sufficiently large wave-speeds for the original reaction-diffusion system and to determine the minimal speed. We prove that for all sufficiently large degradation rates the minimal speed is identical to the minimal speed of the limit problem. In particular, in this parameter range, nonlinear selection of the minimal speed occurs.
\end{abstract}

\section{Contents}

1. Introduction

2. Existence of monotone travelling waves

3. The Stefan-like limit problem

4. Selection of the minimal speed

4.1. Behaviour at infinity

4.2. The reduced system for $c=c_{\infty}$

4.3. Analysis of the decay-rates at $+\infty \quad 17$

4.4. The nonlinear selection regime 19

5. Travelling waves in the fast-degradation-rate limit 20

6. Conclusions 22

6.1. Slow and fast decay at the threshold $c=c_{\infty} \quad 22$

6.2. Discussion 23

References $\quad 24$

\section{INTRODUCTION}

In this article we continue our mathematical analysis of a model for the degradation of host tissue by extracellular bacteria. This model was introduced in $\mathrm{KKC}^{+} 03$ and consists of a reaction-diffusion equation coupled with an ordinary

Date: March 12, 2021.

2000 Mathematics Subject Classification. Primary 35K57; Secondary 92E20, 35B25, 80A22.

Key words and phrases. Travelling waves, Reaction-Diffusion system, singular limit, Stefan problem, pulled fronts, pushed fronts.

The research of the authors was supported by the European Community's Human Potential Programme under contract HPRN-CT-2002-00274, FRONTS-SINGULARITIES. . 
differential equation. In HKR07 we proved the existence of solutions to the time-dependent problem and the convergence to a limit problem in the 'largedegradation-rate' limit. Here we turn to the question of existence and behaviour of travelling-wave solutions.

There is an increasing interest in models which support the understanding of bacterial infections, and we refer to $\left[\mathrm{KKC}^{+} 03\right.$ and $\left.\mathrm{WKK}^{+} 04\right]$ for further background and references. This paper is in effect concerned with the specific issue of how rapidly a bacterial infection in, for example, a burn wound may invade the underlying tissue (with dire potential consequences for the patient, notably mortality due to septicemia). For the type of model with which we are concerned here, the relevant invasion speed is expected to be governed by the corresponding travelling-wave problem. Typically, the smallest possible wave-speed is realized by a large class of solutions. Accordingly determining the minimal speed of travelling waves becomes a central question (with obvious implications for the amount of time available for medical treatment, for instance).

In a dimensionless form, the model in $\left[\mathrm{KKC}^{+} 03\right.$ is given by the equations

$$
\begin{aligned}
\partial_{t} u & =\Delta u-u+w-\gamma k u(1-w), \\
\partial_{t} w & =k u(1-w),
\end{aligned}
$$

where $u$ describes the concentration of degradative enzymes, $(1-w)$ the volume fraction of healthy tissue and $\gamma, k$ are positive constants. The key parameter here is the degradation-rate $k$, which is very large in practice. Equations (1.1), (1.2) are considered in a time-space cylinder, with the upper half space of $\mathbb{R}^{3}$ as the spatial domain. Finally, the system is complemented by initial conditions for $u$ and $w$, a Neumann condition on the lateral boundary for $u$ and a decay condition for $u$ and $w$ in the far field. In HKR07 we gave a precise mathematical formulation and proved the existence and uniqueness of solutions to a slightly more general system, including the possibility of a diffusion term in (1.2). One noteworthy aspect of (1.1), (1.2) is the convergence of solutions to the solution of a Stefan-like free boundary problem as the degradation rate $k$ tends to infinity. This large-degradation-rate limit was identified by a formal asymptotic analysis in $\left[\mathrm{KKC}^{+} 03\right.$ and was proved in HKR07.

Reaction-diffusion systems of the general form

$$
V_{t}=D \Delta V+F(V) \text {, }
$$

where $V:(0, T) \times \mathbb{R}^{n} \rightarrow \mathbb{R}^{N}$ is vector-valued, $F: \mathbb{R}^{N} \rightarrow \mathbb{R}^{N}$ is a given nonlinearity and $D \in \mathbb{R}^{N \times N}$ is a diagonal positive-semi-definite matrix, appear in a lot of different scientific areas. One-dimensional travelling waves are solutions on $(0, \infty) \times$ $\mathbb{R}$ of the special form

$$
\tilde{V}(t, x)=V(x-c t),
$$

where $c$ is called the speed and $V$ the profile of this travelling wave. The question of existence and behaviour of travelling waves is of enormous interest in many of the applications and pertinent results for the vector case $N \geq 2$ remain restricted to rather specific systems.

The system (1.1), (1.2) has, as we will see in Remark 2.1, one stable equilibrium in $(u, w)=(1,1)$ and one unstable equilibrium in $(u, w)=(0,0)$ and therefore belongs to the class of monostable systems. Scalar monostable equations

$$
v_{t}=\Delta v+f(v)
$$


where

$$
f(0)=f(1)=0, \quad f(v)>0 \text { for } 0<v<1, \quad f^{\prime}(0)>0>f^{\prime}(1)
$$

are well-studied, especially the famous Fisher equation, that is (1.4) with $f(v)=$ $v(1-v)$, introduced in [Fis37]. The rigorous analysis of equations of this type also started in the 1930s with the work of Kolmogorov, Petrovskii and Piskunov KPP37. Under an extra assumption on $f$ they proved the existence of travelling waves for all speeds $c \geq c_{\text {lin }}$, where $c_{\text {lin }}$ can be found explicitly in terms of $f$ by a linearisation about $v=0$ (corresponding to a degenerate node in the travelling-wave phase plane). Moreover, they proved that the solutions to (1.4) with initial data decaying sufficiently fast propagate with speed $c_{\text {lin }}$. For more general monostable $f$ the propagation speed was found to be either equal to or larger than $c_{\text {lin }}$ and therefore one distinguishes between a linear or nonlinear selection of the propagation speed (the terminology pulled and pushed fronts, respectively, having an equivalent meaning). Aronson and Weinberger AW75 (see also Hadeler and Rothe HR75. and Sto76 for other pioneering work on such matters and vS03 for a recent review) proved that for general monostable $f$, in both the linear and the nonlinear selection case, the propagation speed for solutions with initial data decaying sufficiently fast is given by the minimal speed of travelling waves. They showed that monotonic travelling waves exist for all speeds $c \geq c_{\min }$ and none for $c<c_{\min }$, where $c_{\min } \geq c_{\text {lin }}$; solutions of (1.4) with sufficiently rapidly decaying initial data propagate with speed $c_{\min }$. For the nonlinear selection cases, $c_{\min }>c_{\text {lin }}$, Rothe Rot81 and Roquejoffre Roq97 proved that, if the initial data decays sufficiently rapidly, the large-time solutions to (1.4) not only propagate with speed $c_{\min }$ but also approach the profile of a travelling wave with minimal speed.

Whereas the connection between large-time behaviour and existence of travelling waves for monostable equations is satisfactorily resolved, the distinction between nonlinear or linear selection is still a challenging question, see for example vS89], EvS00, BD94. Only a few rigorous results for general monostable nonlinearities are available. In LMN04 a variational characterisation of travelling waves and a concrete criterion for whether linear or nonlinear selection occurs for a given equation was derived. Even fewer general analytical results are available for monostable systems. An existence theorem for travelling waves was proved in [VVV94 for monotone monostable systems, which are systems of the form (1.3) in which the Jacobian matrix $D F$ has only nonnegative off-diagonal elements. Results on the existence of travelling waves and the long time behaviour of (1.3) for monostable gradient systems, that is for $n=N$ and nonlinearities $F=\nabla g$ with $g: \mathbb{R}^{n} \rightarrow \mathbb{R}$, were given in Mur04. To the best of our knowledge there are no more general results on the question of whether linear or nonlinear selection will occur.

In this article we prove that for all $\gamma, k$ there exist monotone travelling waves for the system (1.1), (1.2) for all speeds $c \geq c_{\min }$ and no speeds $c<c_{\min }$. The minimal speed $c_{\min }$ in general depends on the parameters $\gamma, k$.

We prove that for all $k>k_{0}$, where $k_{0}$ is explicitly given in terms of $\gamma$, the minimal speed is larger than the value $c_{\text {lin }}(k)$ obtained from a linearisation at the unstable equilibrium. Surprisingly enough, for $k>k_{0}$ the minimal speed of travelling waves is identical to the minimal speed of travelling waves for the Stefanlike limit problem that was formulated in [HKR07]. Our analysis is based on two main facts. One is the monotone structure of the system, which makes possible the use of comparison principles for the parabolic problem. The second is a remarkable 
reduction in order of the travelling-wave equations that occurs when $c$ is given by the minimal speed $c_{\infty}$ of travelling waves of the large-degradation limit. We obtain the existence of travelling waves with speed $c_{\infty}$ for (1.1), (1.2). Finally, a comparison argument allows us to prove that nonlinear selection occurs for sufficiently large values of $k$, the minimal speed in this regime being identical to $c_{\infty}$.

This paper is organised as follows. In section 2 we prove the existence of travelling waves for the reaction-diffusion system (1.1), (1.2). In section 3 we recall the formulation of the large degradation limit problem and consider travelling-wave solutions for this problem. In section 4 we return to the reaction-diffusion system and investigate the selection of the minimal speed. Section 5 deals with the convergence of travelling waves for the reaction-diffusion system to travelling waves of the Stefan-like free boundary problem as the reaction rate $k$ approaches infinity. Finally, we give some conclusions and remarks on open problems in section 6 .

\section{EXISTENCE OF MONOTONE TRAVELLING WAVES}

In this section we prove the existence of monotone travelling waves. First we fix some notation and make some remarks.

Remark 2.1. A system of the general form (1.3) is called monotone if the offdiagonal elements of the Jacobian matrix DF are non-negative and strictly monotone if they are positive (see [VVV94). A system of the form (1.3) with two stationary points is called monostable if one of the stationary points is stable and the other is unstable.

The system (1.1), (1.2) is of the form (1.3) with

$$
V=\left(\begin{array}{c}
u \\
w
\end{array}\right), \quad F\left(z_{1}, z_{2}\right)=\left(\begin{array}{c}
-z_{1}+z_{2}-\gamma k z_{1}\left(1-z_{2}\right) \\
k z_{1}\left(1-z_{2}\right)
\end{array}\right) .
$$

It follows that (1.1), (1.2) is a monotone but not strictly monotone system. Further, $z=(0,0)$ and $z=(1,1)$ are the only stationary points of (1.1), (1.2) and we obtain

$$
D F(0,0)=\left(\begin{array}{cc}
-(1+\gamma k) & 1 \\
k & 0
\end{array}\right)
$$

with one positive and one negative eigenvalue, and

$$
D F(1,1)=\left(\begin{array}{cc}
-1 & 1+\gamma k \\
0 & -k
\end{array}\right)
$$

with two negative eigenvalues. Therefore (1.1), (1.2) is a monostable monotone system.

As remarked before, for a one-dimensional travelling wave $u, w$ of (1.1), (1.2) with speed $c$, the functions

$$
\tilde{u}(t, x):=u(x-c t), \quad \tilde{w}(t, x):=w(x-c t),
$$

are solutions of (1.1), (1.2) on $(0, \infty) \times \mathbb{R}$. Therefore $u, w$ have to satisfy the travelling-wave equations

$$
\begin{aligned}
& 0=u^{\prime \prime}+c u^{\prime}-u+w-\gamma k u(1-w), \\
& 0=c w^{\prime}+k u(1-w),
\end{aligned}
$$

We restrict our investigations to functions $u, w$ taking values only in $[0,1]$, which is the range of meaningful values in the tissue degradation model. We first summarise some basic properties of travelling-wave solutions. 
Lemma 2.2. Assume that $u \in C^{2}(\mathbb{R}), w \in C^{1}(\mathbb{R})$ with $0 \leq u, w \leq 1$ satisfy (2.1), (2.2) with $c \in \mathbb{R}$. Then the following properties hold.

(1) Invariance under space shifts. For any $x_{0} \in \mathbb{R}$ the functions defined by $x \mapsto$ $u\left(x+x_{0}\right), x \mapsto w\left(x+x_{0}\right)$ solve (2.1), (2.2) with the same $c \in \mathbb{R}$.

(2) Invariance under inversion. The functions defined by $x \mapsto u(-x), x \mapsto w(-x)$ solve (2.1), (2.2) with $c$ replaced by $-c$.

(3) Smoothness of travelling waves. The functions $u, w$ are infinitely differentiable.

(4) Monotonicity of travelling waves. If $c>0$ and $u, w$ are not both constant then $u, w$ are strictly monotone decreasing and they approach unity as $x \rightarrow-\infty$ and zero as $x \rightarrow \infty$.

(5) Standing waves. For $c=0$ either $(u, w)=(0,0)$ or $(u, w)=(1,1)$.

Proof. The statements (1), (2) are immediate from (2.1), (2.2). Statement (3) follows from a bootstrapping argument.

To prove (4) we observe from (2.2) that $w^{\prime} \leq 0$ and $w^{\prime}<0$ if $u(1-w)>0$. Next we differentiate (2.1) and obtain

$$
0=u^{\prime \prime \prime}+c u^{\prime \prime}-(1+\gamma k(1-w)) u^{\prime}+(1+\gamma k u) w^{\prime} .
$$

From this equation we see that $u^{\prime}$ cannot have a positive local maximum. Since $u$ is bounded, $u^{\prime}$ cannot approach a positive supremum at $+\infty$ or $-\infty$ and we obtain that $u^{\prime} \leq 0$.

Assume now that $u^{\prime}\left(x_{0}\right)=0$. Therefore $u^{\prime}\left(x_{0}\right)$ is a local maximum of $u^{\prime}$ and we deduce from (2.3) that $w^{\prime}\left(x_{0}\right) \geq 0$ and that, using $w^{\prime} \leq 0$ and (2.2),

$$
-\frac{k}{c} u(1-w)\left(x_{0}\right)=w^{\prime}\left(x_{0}\right)=0 .
$$

Let us consider the case $u\left(x_{0}\right)=0$ (the case that $w$ takes the value one is analogous). Then, by $u^{\prime} \leq 0$ and $u \geq 0$, we obtain $u=0$ on $\left[x_{0}, \infty\right)$. From (2.1) it also follows that $w=0$ on $\left[x_{0}, \infty\right)$. Since $u, w$ are smooth we find that $u, w$ solve (2.1), (2.2) with $u\left(x_{0}\right)=u^{\prime}\left(x_{0}\right)=w\left(x_{0}\right)=0$. On the other hand solutions of (2.1), (2.2) with these data prescribed at $x_{0}$ are unique and thus identically equal to zero, in contradiction to our assumption of non-constant solutions. Therefore $u(1-w)>0$ and $u^{\prime}, w^{\prime}<0$ holds.

Next, since $u(x), w(x)$ are monotone in $x$ and uniformly bounded, their limits as $x \rightarrow-\infty$ exist. From (2.2) we deduce that $w^{\prime}(x)$ has a limit and, from $0 \leq w \leq 1$, that

$$
w^{\prime}(x) \rightarrow 0, \quad u(x)(1-w(x)) \rightarrow 0 \quad \text { as } x \rightarrow-\infty .
$$

From (2.1) we similarly obtain that

$$
\left(u^{\prime}+c u\right)^{\prime}(x) \rightarrow 0 \quad \text { as } x \rightarrow-\infty,
$$

and thus

$$
-u(x)+w(x) \rightarrow 0 \quad \text { as } x \rightarrow-\infty .
$$

By $0 \leq u, w \leq 1$, (2.4), and $u^{\prime}, w^{\prime} \leq 0$ this gives

$$
u(x) \rightarrow 1, \quad w(x) \rightarrow 1 \quad \text { as } x \rightarrow-\infty .
$$

The proof that $u(x), w(x)$ approach zero as $x \rightarrow+\infty$ is similar.

To prove claim (5) we first deduce from $c=0$ and (2.2) that

$$
u(1-w)=0 .
$$


Let us assume that $u\left(x_{0}\right)>0$ for a point $x_{0} \in \mathbb{R}$. Then there exists a maximal interval $(a, b)$ such that $x_{0} \in(a, b)$ and $u>0$ in $(a, b)$. From (2.1), (2.5), and $c=0$ we then obtain that

$$
u>0, w=1, u^{\prime \prime}<0 \quad \text { in }(a, b) .
$$

If $a>-\infty$ we deduce from the regularity assumptions on $u, w$ and $u \geq 0$ that

$$
u(a)=0, w(a)=1, u^{\prime}(a)=0
$$

and finally $u^{\prime \prime}(a) \geq 0$, which yields a contradiction to (2.1). This shows that $a=-\infty$; by analogous arguments we obtain that $b=\infty$ and $(a, b)=\mathbb{R}$. Therefore

$$
0=u^{\prime \prime}-u+1 \text { on } \mathbb{R} \text {. }
$$

and from the boundedness of $u$ it follows that $u=1=w$.

Similarly we prove that $w=0=u$ if there exists $x_{0} \in \mathbb{R}$ such that $w\left(x_{0}\right)<1$.

Due to Lemma 2.2 we can restrict our investigations to the following set of admissible functions.

Definition 2.3. Let $\mathcal{K}$ be the set of functions $v \in C^{\infty}(\mathbb{R})$, with

$$
\begin{gathered}
0<v<1, \quad v^{\prime}<0, \\
v(x) \rightarrow 1, \quad \text { as } x \rightarrow-\infty, \\
v(x) \rightarrow 0, \quad \text { as } x \rightarrow+\infty .
\end{gathered}
$$

$W e$ call $(c, u, w) \in \mathbb{R} \times \mathcal{K} \times \mathcal{K}$ satisfying (2.1), 2.2) a monotone (decreasing) travelling wave for (1.1), (1.2).

We observe from (2.2) that the speed of a monotone (decreasing) travelling wave is always positive.

Theorem 2.4 (Existence of travelling waves). For all $\gamma, k$ there is a positive number $c_{\min }<\infty, c_{\min }=c_{\min }(\gamma, k)$, such that there exists a monotone travelling wave of (1.1), (1.2) with speed $c$ for all $c \geq c_{\min }$ and such that there is no monotone travelling wave with speed $c<c_{\min }$.

The value $c_{\text {min }}$ gives thus the minimal speed of travelling waves for (1.1), (1.2).

In the remainder of this section we prove Theorem 2.4 .

For $0<\varepsilon<(2 \gamma)^{-1}$ we consider the following strictly monotone and strictly parabolic approximation of (1.1), (1.2),

$$
\begin{aligned}
& \partial_{t} \tilde{u}_{\varepsilon}=\partial_{x x} \tilde{u}_{\varepsilon}-\tilde{u}_{\varepsilon}+\tilde{w}_{\varepsilon}-\gamma k \tilde{u}_{\varepsilon}\left(1-\tilde{w}_{\varepsilon}\right), \\
& \partial_{t} \tilde{w}_{\varepsilon}=\varepsilon \partial_{x x} \tilde{w}_{\varepsilon}+\varepsilon\left(\tilde{u}_{\varepsilon}-\tilde{w}_{\varepsilon}\right)+k \tilde{u}_{\varepsilon}\left(1-\tilde{w}_{\varepsilon}\right),
\end{aligned}
$$

and the corresponding travelling-wave equations

$$
\begin{aligned}
& 0=u_{\varepsilon}^{\prime \prime}+c u_{\varepsilon}^{\prime}-u_{\varepsilon}+w_{\varepsilon}-\gamma k u_{\varepsilon}\left(1-w_{\varepsilon}\right), \\
& 0=\varepsilon w_{\varepsilon}^{\prime \prime}+c w_{\varepsilon}^{\prime}+\varepsilon\left(u_{\varepsilon}-w_{\varepsilon}\right)+k u_{\varepsilon}\left(1-w_{\varepsilon}\right) .
\end{aligned}
$$

Observe that the ODE system (2.12), (2.13) has the same stationary points as the original system (2.1), (2.2). For $\varepsilon=0$ the systems (2.12), (2.13) and (2.1), (2.2) coincide. The existence of travelling waves for the auxiliary problem and a 
variational characterisation of the minimal speed follows from VVV94. To state their result, we define functionals $\Phi_{1}^{\varepsilon}, \Phi_{2}^{\varepsilon}: \mathcal{K} \times \mathcal{K} \rightarrow \mathbb{R}$,

$$
\begin{aligned}
\Phi_{1}^{\varepsilon}(\varrho, \sigma) & :=\sup _{x \in \mathbb{R}} \frac{\varrho^{\prime \prime}(x)-\varrho(x)+\sigma(x)-\gamma k \varrho(x)(1-\sigma(x))}{-\varrho^{\prime}(x)}, \\
\Phi_{2}^{\varepsilon}(\varrho, \sigma) & :=\sup _{x \in \mathbb{R}} \frac{\varepsilon \sigma^{\prime \prime}(x)+\varepsilon(\varrho-\sigma)(x)+k \varrho(x)(1-\sigma(x))}{-\sigma^{\prime}(x)} .
\end{aligned}
$$

Lemma 2.5 ([VVV94] Theorem I.4.2). For all $c \geq c_{\varepsilon}$, where $c_{\varepsilon} \geq 0$ is defined by

$$
c_{\varepsilon}:=\inf _{\sigma, \varrho \in \mathcal{K}} \max \left(\Phi_{1}^{\varepsilon}(\varrho, \sigma), \Phi_{2}^{\varepsilon}(\varrho, \sigma)\right),
$$

there exists a monotone travelling wave $\left(c, u_{\varepsilon}, w_{\varepsilon}\right)$ and $c_{\varepsilon}$ is the minimal speed of travelling waves for (2.10), (2.11).

To prove the existence of travelling waves for (1.1), (1.2) we pass in (2.12), (2.13) to the limit $\varepsilon \rightarrow 0$. First we derive bounds for $u_{\varepsilon}, w_{\varepsilon}$ which are uniform in $\varepsilon>0$.

Lemma 2.6. Consider $\varepsilon>0$ and let $\left(c, u_{\varepsilon}, w_{\varepsilon}\right)$ be a monotone travelling wave for (2.10), (2.11). Define $\mu, \nu>0$ to be the positive solutions of

$$
\begin{aligned}
\mu^{2}+c \mu-1 & =0, \\
\nu^{2}-c \nu-(1+\gamma k) & =0 .
\end{aligned}
$$

Then

$$
\begin{aligned}
u_{\varepsilon}^{\prime} & \geq-\mu\left(1-u_{\varepsilon}\right), \\
u_{\varepsilon}^{\prime} & \geq-\nu u_{\varepsilon} \\
\left|u_{\varepsilon}^{\prime \prime}\right| & \leq c \mu+1+\gamma k \\
w_{\varepsilon}^{\prime} & \geq-\frac{\varepsilon+k}{c}\left(1-w_{\varepsilon}\right)
\end{aligned}
$$

hold.

Proof. We fix $x \in \mathbb{R}$ and define for $y \in(-\infty, x]$

$$
\begin{aligned}
& \hat{u}(y):=1-\left(1-u_{\varepsilon}(x)\right) e^{\mu(y-x)}, \\
& \hat{w}(y):=1-\left(1-w_{\varepsilon}(x)\right) e^{L(y-x)}, \quad \text { where } L:=\frac{k+\varepsilon}{c} .
\end{aligned}
$$

Then we observe that

$$
\begin{aligned}
\lim _{y \rightarrow-\infty} \hat{u}(y)=1, & \hat{u}(x)=u_{\varepsilon}(x), \\
\lim _{y \rightarrow-\infty} \hat{w}(y)=1, & \hat{w}(x)=w_{\varepsilon}(x) .
\end{aligned}
$$

Moreover

$$
\begin{gathered}
\hat{u}^{\prime \prime}+c \hat{u}^{\prime}-\hat{u}+w_{\varepsilon}-\gamma k \hat{u}\left(1-w_{\varepsilon}\right) \\
=-\left(1-u_{\varepsilon}(x)\right)\left(\mu^{2}+c \mu-1\right) e^{\mu(y-x)}-\left(1-w_{\varepsilon}\right)-\gamma k \hat{u}\left(1-w_{\varepsilon}\right)<0
\end{gathered}
$$

and

$$
\begin{gathered}
\varepsilon \hat{w}^{\prime \prime}+c \hat{w}^{\prime}-\varepsilon \hat{w}+\varepsilon u_{\varepsilon}+k u_{\varepsilon}(1-\hat{w}) \\
=-\left(1-w_{\varepsilon}(x)\right)\left(\varepsilon L^{2}+c L-\left(\varepsilon+k u_{\varepsilon}\right)\right) e^{L(y-x)}-\varepsilon\left(1-u_{\varepsilon}\right)(x)<0 .
\end{gathered}
$$


By the maximum principle, which we apply once for the scalar equation (2.12) and once for the scalar equation (2.13), we deduce that

$$
u_{\varepsilon} \leq \hat{u}, \quad w_{\varepsilon} \leq \hat{w} \quad \text { on }(-\infty, x],
$$

thus

$$
\begin{aligned}
u_{\varepsilon}^{\prime}(x) & \geq \hat{u}^{\prime}(x)=-\mu\left(1-u_{\varepsilon}(x)\right), \\
w_{\varepsilon}^{\prime}(x) & \geq \hat{w}^{\prime}(x)=-L\left(1-w_{\varepsilon}(x)\right),
\end{aligned}
$$

which proves (2.17), (2.20). The estimate (2.19) follows from (2.12) and (2.17). In addition, by comparing $u_{\varepsilon}$ on $[x, \infty)$ with

$$
\check{u}(y):=u_{\varepsilon}(x) e^{-\nu(y-x)}
$$

we obtain

$$
\begin{aligned}
\check{u} & \leq u_{\varepsilon} \text { on }[x, \infty), \\
\check{u}^{\prime}(x) & \leq u_{\varepsilon}^{\prime}(x),
\end{aligned}
$$

which yields (2.18).

By similar arguments one proves that corresponding properties hold for travelling-wave solutions of (1.1), (1.2).

Lemma 2.7. Let $(c, u, w)$ be a monotone travelling wave for (1.1), (1.2). Define $\mu, \nu>0$ to be the positive solutions of

$$
\begin{aligned}
\mu^{2}+c \mu-1 & =0, \\
\nu^{2}-c \nu-(1+\gamma k) & =0 .
\end{aligned}
$$

Then

$$
\begin{aligned}
u^{\prime} & \geq-\mu(1-u), \\
u^{\prime} & \geq-\nu u, \\
\left|u^{\prime \prime}\right| & \leq c \mu+1+\gamma k, \\
w^{\prime} & \geq-\frac{k}{c}(1-w)
\end{aligned}
$$

hold.

We prove now the first statement in Theorem 2.4.

Proposition 2.8. For each $c \geq c_{0}$, where

$$
c_{0}:=\liminf _{\varepsilon \rightarrow 0} c_{\varepsilon}
$$

there exists a monotone travelling wave $(c, u, w)$ for (1.1), (1.2). Moreover, the value $c_{0}$ is finite.

Proof. Assume first that $c_{0}<\infty$ and fix an arbitrary $c \geq c_{0}$ and a subsequence $\varepsilon_{i} \rightarrow 0(i \rightarrow \infty)$ such that

$$
c_{0}=\lim _{i \rightarrow \infty} c_{\varepsilon_{i}} .
$$

By Lemma 2.5 there exists a sequence of monotone travelling waves $\left(c_{i}, u_{\varepsilon_{i}}, w_{\varepsilon_{i}}\right)$ for (2.10), (2.11), with $c_{i}=c$ if $c>c_{0}$ and $c_{i}=c_{\varepsilon_{i}}$ if $c=c_{0}$ such that

$$
\begin{aligned}
0 & =u_{\varepsilon_{i}}^{\prime \prime}+c_{i} u_{\varepsilon_{i}}^{\prime}-u_{\varepsilon_{i}}+w_{\varepsilon_{i}}-\gamma k u_{\varepsilon_{i}}\left(1-w_{\varepsilon_{i}}\right), \\
0 & =\varepsilon_{i} w_{\varepsilon_{i}}^{\prime \prime}+c_{i} w_{\varepsilon_{i}}^{\prime}+\varepsilon_{i}\left(u_{\varepsilon_{i}}-w_{\varepsilon_{i}}\right)+k u_{\varepsilon_{i}}\left(1-w_{\varepsilon_{i}}\right) .
\end{aligned}
$$


Since travelling waves are invariant under space shifts, we can assume without loss of generality that

$$
u_{\varepsilon_{i}}(0)=\frac{1}{2} \quad \text { for all } i \in \mathbb{N} .
$$

By $0<u_{\varepsilon_{i}}, w_{\varepsilon_{i}}<1$, the monotonicity of $w_{\varepsilon_{i}}$ and (2.17), (2.19) there exists $u \in$ $C^{1,1}(\mathbb{R}), w \in L^{\infty}(\mathbb{R})$ such that for all $0<\alpha<1, R>0$

$$
\begin{aligned}
u_{\varepsilon_{i}} & \rightarrow u \quad \text { in } C^{1, \alpha}([-R, R]), \\
w_{\varepsilon_{i}} & \rightarrow w \quad \text { pointwise almost everywhere in } \mathbb{R}
\end{aligned}
$$

hold for a subsequence $\varepsilon_{i} \rightarrow 0(i \rightarrow \infty)$.

Multiplying (2.29), (2.30) by a function $\eta \in C_{c}^{\infty}(\mathbb{R})$ and integrating we deduce

$$
\begin{aligned}
0 & =\int_{\mathbb{R}}\left(-\eta^{\prime} u_{\varepsilon_{i}}^{\prime}+c_{i} \eta u_{\varepsilon_{i}}^{\prime}-\eta\left(u_{\varepsilon_{i}}-w_{\varepsilon_{i}}\right)-\eta \gamma k u_{\varepsilon_{i}}\left(1-w_{\varepsilon_{i}}\right)\right), \\
0 & =\int_{\mathbb{R}}\left(\varepsilon_{i} \eta^{\prime \prime} w_{\varepsilon_{i}}-c_{i} \eta^{\prime} w_{\varepsilon_{i}}+\varepsilon_{i} \eta\left(u_{\varepsilon_{i}}-w_{\varepsilon_{i}}\right)+k \eta u_{\varepsilon_{i}}\left(1-w_{\varepsilon_{i}}\right)\right) .
\end{aligned}
$$

Due to (2.32), (2.33) we can pass to the limit $\varepsilon_{i} \rightarrow 0$ in these equations and get

$$
\begin{aligned}
& 0=\int_{\mathbb{R}}\left(-\eta^{\prime} u^{\prime}+c \eta u^{\prime}-\eta(u-w)-\eta \gamma k u(1-w)\right), \\
& 0=\int_{\mathbb{R}}\left(-c \eta^{\prime} w+k \eta u(1-w)\right) .
\end{aligned}
$$

It follows that $(c, u, w)$ solve (2.1), (2.2) and, by a bootstrapping argument, that $u, w$ are smooth. Moreover, (2.31) and (2.32) yield that $u(0)=1 / 2$ and by Lemma 2.2 we obtain that $c>0$ and that $(c, u, w)$ is a monotone travelling wave. Since $c \geq c_{0}$ was arbitrary, the first part of the Proposition is proved. To prove that $c_{0}<\infty$, let $\varrho, \sigma$ be two smooth strictly monotonically decreasing functions with

$$
\begin{array}{ll}
\varrho(x)=\sigma(x)=e^{-x} & \text { for all } x \geq 1, \\
\varrho(x)=\sigma(x)=1-e^{-x} & \text { for all } x \leq-1 .
\end{array}
$$

Then there exists a constant $C(k)<\infty$ such that for all $0<\varepsilon<1$ and all $|x| \geq 1$

$$
\begin{aligned}
& \frac{\varrho^{\prime \prime}(x)-\varrho(x)+\sigma(x)-\gamma k \varrho(x)(1-\sigma(x))}{-\varrho^{\prime}(x)} \leq C(k), \\
& \frac{\varepsilon \sigma^{\prime \prime}(x)+\varepsilon(\varrho-\sigma)(x)+k \varrho(x)(1-\sigma(x))}{-\sigma^{\prime}(x)} \leq C(k) .
\end{aligned}
$$

We can estimate the same ratios for all $0<\varepsilon<1$ and $|x| \leq 1$ by a constant depending only on $\|\varrho\|_{C^{2}([-1,1])},\|\sigma\|_{C^{2}([-1,1])}$. By Lemma 2.5] and the definition of $c_{\varepsilon}$ in (2.14) it follows that

$$
c_{\varepsilon} \leq C(k) \quad \text { for all } 0<\varepsilon<1 .
$$

In particular, $c_{0} \leq C(k)$ is finite.

We now complete the proof of Theorem 2.4 .

Proposition 2.9. There is no monotone travelling wave for (1.1), (1.2) with speed $c<c_{0}$. 
Proof. Assume that $(c, u, w)$ with $c<c_{0}, u, w \in \mathcal{K}$ satisfies (2.1), (2.2). We then obtain that

$$
\begin{aligned}
& \Phi_{1}^{\varepsilon}(u, w)=c, \\
& \Phi_{2}^{\varepsilon}(u, w) \leq c+\varepsilon \sup _{x \in \mathbb{R}} \frac{w^{\prime \prime}(x)+u(x)-w(x)}{-w^{\prime}(x)} .
\end{aligned}
$$

From (2.2) and $u<1$ we deduce that

$$
\frac{u-w}{-w^{\prime}} \leq \frac{u(1-w)}{-w^{\prime}}=\frac{c}{k} .
$$

A differentiation in (2.2) yields that

$$
-c w^{\prime \prime}=k u^{\prime}(1-w)-k u w^{\prime}=-c w^{\prime} \frac{u^{\prime}}{u}-k u w^{\prime}
$$

which gives, together with (2.25), that

$$
\frac{w^{\prime \prime}}{-w^{\prime}}=\frac{-u^{\prime}}{u}-\frac{k}{c} u \leq \nu
$$

with $\nu$ as in (2.23). Using (2.35), (2.36) in (2.34) we obtain that

$$
\Phi_{2}^{\varepsilon}(u, w) \leq c+\varepsilon C,
$$

where $C$ is independent of $\varepsilon>0$. From Lemma 2.5 and the definition of $c_{0},(2.14)$ and (2.28) we deduce that

$$
c_{0} \leq \liminf _{\varepsilon \rightarrow 0} \max \left(\Phi_{1}^{\varepsilon}(u, w), \Phi_{2}^{\varepsilon}(u, w)\right)=c,
$$

which is a contradiction to our assumption $c<c_{0}$.

\section{The Stefan-Like Limit Problem}

The reaction-diffusion system (1.1), (1.2) converges to a Stefan-like free boundary problem as $k$ tends to infinity, see HKR07. For solutions $U_{\infty}, W_{\infty}$ of this limit problem

$$
0=U_{\infty}\left(1-W_{\infty}\right)
$$

holds and the spatial domain splits in a region where $U_{\infty}=0$, and a region where $U_{\infty}>0$ and $W_{\infty}=1$. If we denote their common boundary at time $t$ by $\Gamma(t)$ then $U_{\infty}, W_{\infty}$ satisfy

$$
\begin{aligned}
\partial_{t} U_{\infty} & =\Delta U_{\infty}-U_{\infty}+1 & & \text { in }\left\{W_{\infty}=1\right\} \\
\gamma \partial_{t} W_{\infty} & =W_{\infty} & & \text { in }\left\{U_{\infty}=0\right\}
\end{aligned}
$$

and a continuity and jump condition on $\Gamma(t)$,

$$
\begin{aligned}
{\left[U_{\infty}(t, .)\right] } & =0, \\
-\left[\nabla U_{\infty}(t, .) \cdot \nu(t, .)\right] & =\gamma\left[W_{\infty}(t, .)\right] \vec{v}(t, .) \cdot \nu(t, .),
\end{aligned}
$$

where $\vec{v}(t,$.$) and \nu(t,$.$) are the velocity and the unit normal of the free boundary$ $\Gamma(t)$, pointing into $\left\{U_{\infty}=0\right\}$, and [.] denotes the jump across the free boundary from the region $\left\{U_{\infty}>0\right\}$ to $\left\{U_{\infty}=0\right\}$.

As for the reaction-diffusion system, travelling-wave solutions $U_{\infty}, W_{\infty}$ are given by a speed $c \in \mathbb{R}$ and profile functions $u_{\infty}, w_{\infty}$,

$$
U_{\infty}(x, t)=u_{\infty}(x-c t), \quad W_{\infty}(x, t)=w_{\infty}(x-c t) .
$$


We are interested in monotone travelling waves which connect unity and zero,

$$
\begin{aligned}
& u_{\infty}(x), w_{\infty}(x) \rightarrow 1 \quad \text { as } x \rightarrow-\infty \\
& u_{\infty}(x), w_{\infty}(x) \rightarrow 0 \quad \text { as } x \rightarrow \infty \\
& u_{\infty}, w_{\infty} \quad \text { are monotonically decreasing. }
\end{aligned}
$$

Due to the shift invariance of travelling waves, the condition (3.1) and the monotonicity of $u_{\infty}, w_{\infty}$ we can assume that

$$
\begin{array}{ll}
u_{\infty}(x)=0 & \text { for } x \geq 0, \\
u_{\infty}(x)>0, w_{\infty}(x)=1 & \text { for } x<0 .
\end{array}
$$

From 3.2 -3.5 wen obtain

$$
\begin{array}{ll}
0=u_{\infty}^{\prime \prime}+c u_{\infty}^{\prime}-u_{\infty}+1 & \text { for } x<0, \\
0=\gamma c w_{\infty}^{\prime}+w_{\infty} & \text { for } x>0,
\end{array}
$$

and the continuity and jump condition

$$
\begin{aligned}
u_{\infty}(0-) & =0, \\
\gamma c\left(1-w_{\infty}(0+)\right) & =-u_{\infty}^{\prime}(0-) .
\end{aligned}
$$

Proposition 3.1. For all $c \geq c_{\infty}$, where

$$
c_{\infty}:=\frac{1}{\sqrt{\gamma(1+\gamma)}}
$$

there exists a unique solution $u_{\infty}, w_{\infty}$ of (3.6)-(3.14). This solution is given by

$$
\begin{aligned}
& u_{\infty}(x)= \begin{cases}1-e^{\alpha x} & \text { if } x \leq 0, \\
0 & \text { if } x \geq 0\end{cases} \\
& w_{\infty}(x)= \begin{cases}1 & \text { if } x<0, \\
\beta e^{-\frac{x}{c \gamma}} & \text { if } x>0\end{cases}
\end{aligned}
$$

where

$$
\begin{gathered}
\alpha=\alpha(c):=-\frac{c}{2}+\sqrt{\frac{c^{2}}{4}+1}, \\
\beta=\beta(c):=1-\frac{\alpha(c)}{\gamma c} .
\end{gathered}
$$

For $c<c_{\infty}$, there does not exist any solution.

Proof. We deduce that (3.11) and (3.6), (3.13) hold if and only if (3.16) holds, with $\alpha$ as in (3.18). Similarly (3.12) and (3.7) are satisfied if and only if $w_{\infty}$ satisfies (3.17) with $\beta \in \mathbb{R}$. Since

$$
1-w_{\infty}(0+)=1-\beta, \quad-u_{\infty}^{\prime}(0-)=\alpha
$$

we obtain that the jump condition (3.14) is satisfied if and only if $\beta$ satisfies (3.19). Finally, by (3.17) and (3.19), the solution $w_{\infty}$ is nonnegative if and only if $c \geq$ $c_{\infty}$. 


\section{Selection of the minimal Speed}

In this section we prove that for sufficiently large values $k>k_{0}$ a nonlinear selection principle determines the minimal speed of travelling waves. The threshold $k_{0}$ is obtained explicitly in terms of the given constant $\gamma$. First, we have to analyse the behaviour of travelling-wave solutions at infinity.

4.1. Behaviour at infinity. The linear selection principle for the minimal speed is based on the analysis of the linearised system at the unstable stationary point $(u, w)=(0,0)$ of (2.1), 2.2). In the next lemma we show that solutions have to decay exponentially to zero as $x$ tends to infinity.

Lemma 4.1. Let $(c, u, w)$ be a monotone travelling wave. Then

$$
\lim _{x \rightarrow \infty} \frac{1}{x} \log u(x)=\lim _{x \rightarrow \infty} \frac{1}{x} \log w(x)=\lambda,
$$

where $\lambda$ is a negative root of the cubic equation

$$
0=\lambda^{3}+c \lambda^{2}-(1+\gamma k) \lambda-\frac{k}{c} .
$$

Proof. With the definitions

$$
V_{0}(x):=\left(\begin{array}{c}
u^{\prime}(x) \\
u(x) \\
w(x)
\end{array}\right), \quad A_{0}:=\left(\begin{array}{ccc}
-c & 1+\gamma k & -1 \\
1 & 0 & 0 \\
0 & -\frac{k}{c} & 0
\end{array}\right), \quad B_{0}:=\left(\begin{array}{c}
-\gamma \\
0 \\
\frac{1}{c}
\end{array}\right)
$$

the system (2.1), (2.2) is equivalent to

$$
V_{0}^{\prime}(x)=A_{0} V_{0}(x)+k u(x) w(x) B_{0}
$$

and the linearized system at $(0,0,0)$ is given by

$$
\xi^{\prime}(x)=A_{0} \xi(x) .
$$

The eigenvalues of $A_{0}$ are the solutions of (4.2). Since $s \mapsto s^{3}+c s^{2}-(1+\gamma k) s-\frac{k}{c}$ is negative at $s=0$ and becomes positive as $s \rightarrow+\infty$ there exists a positive eigenvalue $\lambda_{+}>0$. The other two solutions of (4.2) satisfy the equation

$$
0=\lambda^{2}+\left(c+\lambda_{+}\right) \lambda+\frac{k}{c \lambda_{+}}
$$

which has, depending on the values of $c, k, \lambda_{+}$, either two negative roots, one repeated negative root or two complex-conjugate roots with negative real part. Since $V_{0}(x)$ converges to zero as $x \rightarrow+\infty$, the curve $x \mapsto V_{0}(x)$ is for sufficiently large values of $x$ contained in the stable manifold and converges exponentially to zero; see for example Per01 Section 2.7. By [CL55] Theorem XIII.4.5 there exists a solution $\xi=\left(\xi_{1}, \xi_{2}, \xi_{3}\right)$ of the linearized system (4.4) and $\delta>0$ with

$$
\begin{aligned}
V_{0}(x) & =\xi(x)+O\left(e^{(\alpha-\delta) x}\right), \\
\xi(x) & =O\left(e^{\alpha x}\right)
\end{aligned}
$$

as $x \rightarrow+\infty$, where $\alpha<0$ is the real part of an eigenvalue $\lambda$ of $A_{0}$. One checks that $A_{0}$ has no eigenvector with a component equal to zero; therefore

$$
\xi_{i}(x)=O\left(e^{\alpha x}\right) \quad \text { for } i=1,2,3
$$

holds as $x \rightarrow+\infty$. Let us show that in fact $\lambda$ is real. Assume that $\lambda=\alpha+i \beta$ with $\beta \neq 0$. Then $\xi(x)$ describes, as $x \rightarrow+\infty$, a spiral around the origin contained in the plane $P$ spanned by the real and imaginary part of an eigenvector of $A_{0}$ with 
eigenvalue $\lambda$. But then, since the difference between $\xi$ and $V_{0}$ decays exponentially faster than $\xi, V_{0}$ has to take values outside the set $\left\{z=\left(z_{1}, z_{2}, z_{3}\right): z_{1}<0, z_{2}>\right.$ $\left.0, z_{3}>0\right\}$, which is a contradiction to the assumption that $(c, u, w)$ is a monotone travelling wave. This shows that $\lambda=\alpha<0$ is real. Thus (4.1) follows from (4.6) and (4.7).

The equation (4.2) connects the speed and the decay rate at $+\infty$ of a travelling wave. We now further analyze this relation.

Lemma 4.2. For all $k>0$ and all $\lambda<0$ there exists a unique value $\bar{c}_{k}(\lambda)>0$ such that $\lambda$ satisfies (4.2) for $c=\bar{c}_{k}(\lambda)$. The function $\lambda \mapsto \bar{c}_{k}(\lambda)$ attains a positive minimum $c_{\operatorname{lin}}=c_{\operatorname{lin}}(k)$ at a unique value $\lambda_{\operatorname{lin}}=\lambda_{\operatorname{lin}}(k)$ and $c_{\operatorname{lin}}, \lambda_{\operatorname{lin}}$ are given by

$$
\begin{aligned}
\lambda_{\operatorname{lin}} & <0 \\
3 \lambda_{\operatorname{lin}}^{2} & =-(1+\gamma k)+2 \sqrt{(1+\gamma k)^{2}+3 k}, \\
\lambda_{\operatorname{lin}} c_{\operatorname{lin}} & =(1+\gamma k)-\sqrt{(1+\gamma k)^{2}+3 k} .
\end{aligned}
$$

Moreover

$$
\begin{array}{ll}
\bar{c}_{k}^{\prime}(\lambda)<0 & \text { for } \lambda<\lambda_{\operatorname{lin}}, \\
\bar{c}_{k}^{\prime}(\lambda)>0 & \text { for } \lambda>\lambda_{\operatorname{lin}},
\end{array}
$$

with

$$
\begin{aligned}
& \bar{c}_{k}(\lambda) \rightarrow \infty \quad \text { as } \lambda \rightarrow-\infty, \\
& \bar{c}_{k}(\lambda) \rightarrow \infty \quad \text { as } \lambda \rightarrow 0 .
\end{aligned}
$$

Proof. From (4.2) we obtain that $\bar{c}_{k}$ is given by

$$
\bar{c}_{k}(\lambda)=-\frac{1}{2}\left(\lambda-(1+\gamma k) \frac{1}{\lambda}\right)+\sqrt{\frac{1}{4}\left(\lambda-(1+\gamma k) \frac{1}{\lambda}\right)^{2}+\frac{k}{\lambda^{2}}},
$$

that $\bar{c}_{k}$ is strictly positive and that $\bar{c}_{k}$ tends to infinity as $\lambda \rightarrow-\infty$ or $\lambda \rightarrow 0$. Therefore the positive minimum $c_{\text {lin }}$ is attained at a value $\lambda_{\operatorname{lin}}$ and $\bar{c}^{\prime}\left(\lambda_{\operatorname{lin}}\right)=0$ holds. By (4.2) this implies

$$
0=3 \lambda_{\operatorname{lin}}^{2}+2 \lambda_{\operatorname{lin}} c_{\operatorname{lin}}-(1+\gamma k) .
$$

Moreover, by (4.2),

$$
0=\lambda_{\operatorname{lin}}^{3}+c_{\operatorname{lin}} \lambda_{\operatorname{lin}}^{2}-(1+\gamma k) \lambda_{\operatorname{lin}}-\frac{k}{c_{\operatorname{lin}}} .
$$

One checks that (4.8), (4.9) is equivalent to (4.12), (4.13). In particular, $\bar{c}_{k}^{\prime}$ has only one zero and we deduce that $\bar{c}_{k}^{\prime}(\lambda)<0$ for $\lambda<\lambda_{\operatorname{lin}}$ and $\bar{c}_{k}^{\prime}(\lambda)>0$ for $0>\lambda>\lambda_{\text {lin }}$.

Corollary 4.3. The minimal speed $c_{\min }(k)$ of travelling waves satisfies the estimate

$$
c_{\min }(k) \geq c_{\text {lin }}(k) .
$$

Proof. According to Lemma 4.1 for a monotone travelling wave with speed $c$, a negative root $\lambda$ of (4.2) exists. On the other hand $c_{\operatorname{lin}}(k)$ gives the minimal value of $c$ such that (4.2) has a negative solution.

We now state the result corresponding to Lemma 4.1 if we consider $x$ approaching $-\infty$. 
Lemma 4.4. Let $(c, u, w)$ be a monotone travelling wave and let $k \geq 1$. Then

$$
\begin{aligned}
\lim _{x \rightarrow-\infty} \frac{1}{x} \log (1-u(x)) & =\mu, \\
\lim _{x \rightarrow-\infty} \frac{1}{x} \log (1-w(x)) & =\frac{k}{c},
\end{aligned}
$$

where $\mu>0$ is given by

$$
\mu=-\frac{c}{2}+\sqrt{\frac{c^{2}}{4}+1} .
$$

For $0<k<1$ the limits in (4.15), (4.16) are either equal to $\mu$ or equal to $k / c$.

Proof. With

$$
V_{1}(x):=\left(\begin{array}{c}
-u^{\prime}(x) \\
1-u(x) \\
1-w(x)
\end{array}\right), \quad A_{1}:=\left(\begin{array}{ccc}
-c & 1 & -(1+\gamma k) \\
1 & 0 & 0 \\
0 & 0 & \frac{k}{c}
\end{array}\right), \quad B_{1}:=\left(\begin{array}{c}
\gamma \\
0 \\
-\frac{1}{c}
\end{array}\right)
$$

the system (2.1), (2.2) is equivalent to

$$
V_{1}^{\prime}(x)=A_{1} V_{1}(x)+k(1-u(x))(1-w(x)) B_{1}
$$

and the linearized system at $V_{1}=(0,0,0)$ is given by

$$
\zeta^{\prime}(x)=A_{1} \zeta(x)
$$

The matrix $A_{1}$ has the positive eigenvalues

$$
\mu=-\frac{c}{2}+\sqrt{\frac{c^{2}}{4}+1}, \quad \mu_{1}=\frac{k}{c}
$$

and one negative eigenvalue

$$
\mu_{2}=-\frac{c}{2}-\sqrt{\frac{c^{2}}{4}+1} .
$$

Since $V_{1}(x) \rightarrow 0$ as $x \rightarrow-\infty$ we deduce that $V_{1}(x)$ is for sufficiently small $x$ contained in the unstable manifold of (4.18) at $(0,0,0)$. Using CL55 Theorem XIII.4.5 we obtain the existence of a solution $\zeta$ of the linearized system (4.19) and a $\delta>0$ with

$$
\begin{aligned}
V_{1}(x) & =\zeta(x)+O\left(e^{(\beta+\delta) x}\right) \\
\zeta(x) & =O\left(e^{\beta x}\right)
\end{aligned}
$$

as $x \rightarrow-\infty$, where $\beta=k / c$ or $\beta=\mu$. One checks that $k / c>\mu$ holds for $k \geq 1$. Thus, if $\beta=k / c$ then the trajectory of $V_{1}$ as $x \rightarrow-\infty$ has to be tangential to the eigenspace of $A_{1}$ corresponding to the eigenvalue $k / c$. On the other hand this eigenspace is spanned by a vector $(k / c, 1, a)$, where $a<0$ for $k \geq 1$. Therefore $V_{1}$ leaves the region $\left\{z=\left(z_{1}, z_{2}, z_{3}\right): z_{2}, z_{3}>0\right\}$, which is a contradiction to $u(x), w(x)<1$. This proves that $\beta=\mu$ for $k \geq 1$ and implies that the trajectory of $\zeta(x)$ for $x \rightarrow-\infty$ is tangential to the eigenspace corresponding to the eigenvalue $\mu$, which is spanned by the vector $(\mu, 1,0)$. Therefore

$$
\zeta_{2}(x)=O\left(e^{\mu x}\right)
$$

and (4.15) follows from (4.20). By (2.2) we deduce

$$
\frac{d}{d x} \log (1-w(x))=\frac{k}{c}+O\left(e^{\mu x}\right) \quad \text { as } x \rightarrow-\infty,
$$


and thus 4.16) holds.

Corollary 4.5. Let $k \geq 1$. If we consider two different monotone travelling waves for (1.1), (1.2), the one with the lower speed converges faster to unity as $x$ approaches $-\infty$.

Proof. For a monotone travelling wave $(c, u, w)$, by Lemma 4.4 the convergence of $u, w$ to unity as $x \rightarrow-\infty$ is exponential with rate $\mu$ for $u$ and rate $k / c$ for $w$, where $\mu$ is given by (4.17). Since

$$
\frac{d}{d c}\left(-\frac{c}{2}+\sqrt{\frac{c^{2}}{4}+1}\right)=\frac{1}{2}\left(-1+\frac{c}{\sqrt{c^{2}+4}}\right)<0
$$

we deduce that both convergence rates are decreasing with $c$.

4.2. The reduced system for $c=c_{\infty}$. It was observed in $\overline{\mathrm{KKC}^{+} 03}$ that the travelling-wave equations (2.1), (2.2) are for the speed $c=c_{\infty}$ defined in (3.15) remarkable in being equivalent to a system of two first-order equations.

Lemma 4.6. Let $u \in \mathcal{K}_{0}, w \in \mathcal{K}$. Then $\left(c_{\infty}, u, w\right)$ is a monotone travelling wave if and only if $u, w$ satisfy

$$
\begin{aligned}
u^{\prime} & =\gamma c_{\infty}(u-w), \\
w^{\prime} & =-k \gamma(1+\gamma) c_{\infty} u(1-w) .
\end{aligned}
$$

Proof. Multiplying (4.23) by $c_{\infty}$ and using (3.15) we see that (4.23) and (2.2) are equivalent for $c=c_{\infty}$. Next we obtain from (3.15) that

$$
\left(u^{\prime}-\gamma c_{\infty}(u-w)\right)^{\prime}=u^{\prime \prime}+c_{\infty} u^{\prime}-\frac{1}{\gamma c_{\infty}} u^{\prime}-\gamma k u(1-w)
$$

and we see that (4.22) implies (2.1). Conversely, from (2.1) and (4.24) we deduce that

$$
\left(u^{\prime}-\gamma c_{\infty}(u-w)\right)^{\prime}=-\frac{1}{\gamma c_{\infty}}\left(u^{\prime}-\gamma c_{\infty}(u-w)\right),
$$

whose solutions are

$$
\left(u^{\prime}-\gamma c_{\infty}(u-w)\right)(x)=a e^{-\frac{1}{\gamma c_{\infty}} x} \quad \text { for any } a \in \mathbb{R} .
$$

The condition that $u(x), w(x)$ converge exponentially to unity as $x \rightarrow-\infty$ implies that $a=0$ and (4.22) therefore holds.

This reduction allows us to prove the existence of a travelling wave with speed $c_{\infty}$ for (1.1), (1.2) by a phase-plane analysis for (4.22), (4.23).

Proposition 4.7. For all $\gamma, k>0$ there exists a monotone travelling wave $\left(c_{\infty}, u_{0}, w_{0}\right)$ for (1.1), (1.2). As $x$ tends to infinity, $u_{0}, w_{0}$ decay exponentially to zero with decay rate

$$
\lambda_{\infty}=\lambda_{\infty}(k):=c_{\infty} \gamma\left(\frac{1}{2}-\sqrt{\frac{1}{4}+k(1+\gamma)}\right)<0
$$

Proof. The system (4.22), (4.23) has the two stationary points $(0,0)$ and $(1,1)$. We define the set

$$
J:=\left\{\left(x_{1}, x_{2}\right)^{T} \in \mathbb{R}^{2}: 0 \leq x_{1}<x_{2} \leq 1\right\}
$$




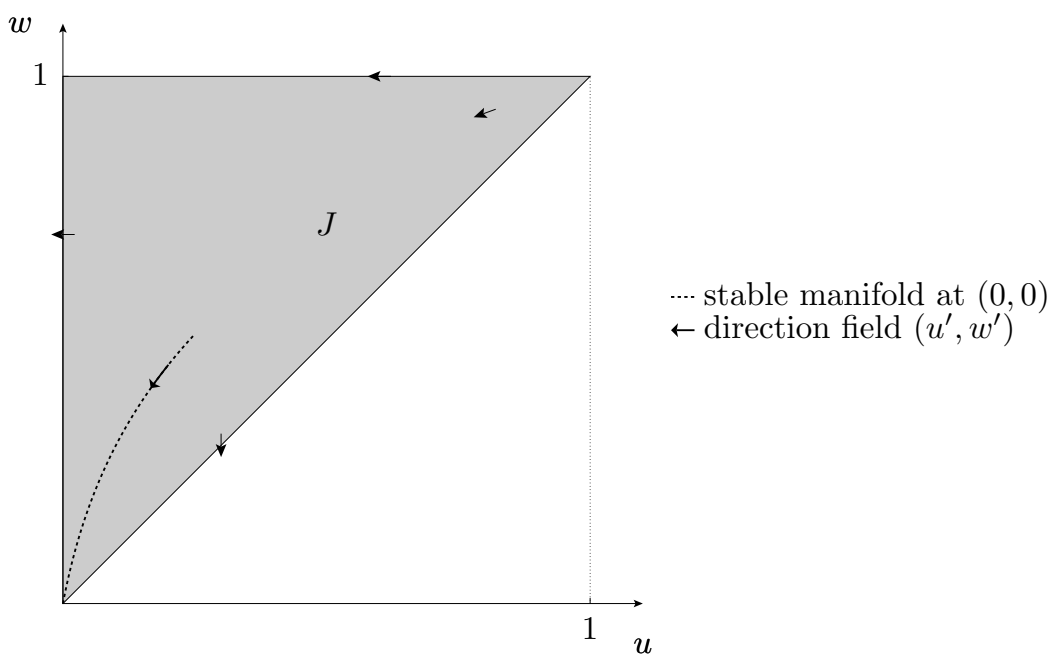

Figure 1. Schematic of the phase portrait of (4.22), (4.23). The dashed area represents the set $J$, the incoming arc the stable manifold at $(0,0)$ and the arrows the direction field.

and observe that $\mathbb{R}^{2} \backslash J$ is an invariant region for (4.22), (4.23) (see Figure 1). Next we consider the linearisation at $(0,0)$ of (4.22), (4.23) which is given by

$$
\zeta^{\prime}=c_{\infty} \gamma\left(\begin{array}{cc}
1 & -1 \\
-k(1+\gamma) & 0
\end{array}\right) \zeta,
$$

for $\xi: \mathbb{R} \rightarrow \mathbb{R}^{2}$. The eigenvalues of this linear system are $\lambda_{\infty}$, as defined in (4.25), and

$$
c_{\infty} \gamma\left(\frac{1}{2}+\sqrt{\frac{1}{4}+k(1+\gamma)}\right)>0 .
$$

An eigenvector with eigenvalue $\lambda_{\infty}$ is given by

$$
v:=\left(\begin{array}{c}
-\frac{1}{2}+\sqrt{\frac{1}{4}+k(1+\gamma)} \\
k(1+\gamma)
\end{array}\right)
$$

One checks that $v_{1}<v_{2}$ holds for the components of $v$ and deduces that the eigenspace corresponding to $\lambda_{\infty}$ intersects the set $J$ defined in (4.26).

By the stable manifold theorem, see for example Per01, Theorem 2.7, there exists a trajectory that, taking $t$ as its parameter variable, converges to $(0,0)$ as $t \rightarrow \infty$ and starts at $t=0$ in $J$, since the stable manifold is tangential to the eigenspace corresponding to the eigenvalue $\lambda_{\infty}$. Following this trajectory back with decreasing $t$ we cannot leave $J$, since otherwise the trajectory would stay in $\mathbb{R}^{2} \backslash J$ as $t$ increases and thus could not reach any point in $J$ at $t=0$. In $J$, with decreasing $t$, the trajectory has to be monotone in both components and therefore has to approach the stationary point $(1,1)$. Thus the trajectory connects $(1,1)$ to $(0,0)$ and satisfies (4.22), (4.23). By Lemma 4.6 this shows that $\left(c_{\infty}, u_{0}, w_{0}\right)$ is a monotone travelling wave for (1.1), (1.2). Moreover $u_{0}(x), w_{0}(x)$ are, for sufficiently large $x$, in the stable manifold of (4.22), (4.23) and we deduce that they converge exponentially fast to zero, with decay rate given by $\lambda_{\infty}$. 
The existence of a travelling wave with speed $c_{\infty}$ implies immediately the following estimate.

Corollary 4.8. The minimal speed $c_{\min }(k)$ of travelling waves satisfies

$$
c_{\min }(k) \leq c_{\infty} .
$$

As we will see, for sufficiently large values of $k$ the minimal speed is identical to the value $c_{\infty}$.

4.3. Analysis of the decay-rates at $+\infty$. In this section we further investigate the deacy of travelling waves to zero as $x$ tends to $+\infty$. With this aim we analyze the functions $\bar{c}_{k}$ defined in Lemma 4.2, $\bar{c}_{k}(\lambda)$ is the speed of a travelling wave with decay rate $\lambda$ at $+\infty$.

Remark 4.9. Corresponding to the reduction (4.22), (4.23) of the travelling-wave system (2.1), (2.2), we find that the equation (4.2) for the possible decay rates factorises for the speed $c_{\infty}=(\gamma(1+\gamma))^{-1 / 2}$. The value

$$
\lambda_{\infty}^{*}:=-\frac{1}{\gamma c_{\infty}}=-\sqrt{\frac{1+\gamma}{\gamma}}
$$

is for all $k>0$ a negative root of (4.2) with $c=c_{\infty}$. The decay rate of $u_{0}, w_{0}$ is given by the other negative root of this equation, which is the value $\lambda_{\infty}(k)$ defined in (4.25).

Lemma 4.10. For $k>0$ consider the values $\lambda_{\operatorname{lin}}(k), c_{\operatorname{lin}}(k)$ as defined in Lemma 4.2 and the values $\lambda_{\infty}(k), \lambda_{\infty}^{*}$ as given in (4.25), (4.28). Then there exists a unique $k_{0}>0$, which is is given explicitely by $k_{0}=(1+2 \gamma) \gamma^{-2}$, such that

$$
\lambda_{\operatorname{lin}}\left(k_{0}\right)=\lambda_{\infty}^{*} .
$$

Moreover, for $0<k_{1}<k_{0}<k_{2}$

$$
\begin{aligned}
& \lambda_{\infty}\left(k_{1}\right)>\lambda_{\operatorname{lin}}\left(k_{1}\right)>\lambda_{\infty}^{*}, \\
& \lambda_{\infty}\left(k_{0}\right)=\lambda_{\operatorname{lin}}\left(k_{0}\right)=\lambda_{\infty}^{*}, \\
& \lambda_{\infty}\left(k_{2}\right)<\lambda_{\operatorname{lin}}\left(k_{2}\right)<\lambda_{\infty}^{*}
\end{aligned}
$$

hold, see Figure 2

Proof. We have proved in Lemma 4.2 that the functions $\bar{c}_{k}$ attain their minimum $c_{\operatorname{lin}}(k)$ at a unique value $\lambda_{\operatorname{lin}}(k)$,

$$
\begin{aligned}
& c_{\operatorname{lin}}(k)=\bar{c}_{k}\left(\lambda_{\operatorname{lin}}(k)\right), \\
& \bar{c}_{k}(\lambda)>c_{\operatorname{lin}}(k) \quad \text { for all } \lambda<0, \lambda \neq \lambda_{\operatorname{lin}}(k) .
\end{aligned}
$$

For convenience we recall that

$$
\begin{aligned}
3 \lambda_{\operatorname{lin}}^{2} & =-(1+\gamma k)+2 \sqrt{(1+\gamma k)^{2}+3 k}, \quad \lambda_{\operatorname{lin}}<0, \\
\lambda_{\operatorname{lin}} c_{\operatorname{lin}} & =(1+\gamma k)-\sqrt{(1+\gamma k)^{2}+3 k} .
\end{aligned}
$$

By Proposition 4.7 Remark 4.9 and the definition of $\bar{c}_{k}$ in Lemma 4.2

$$
\bar{c}_{k}\left(\lambda_{\infty}^{*}\right)=\bar{c}_{k}\left(\lambda_{\infty}(k)\right)=c_{\infty} \quad \text { for all } k>0
$$

holds. Next we see from (4.35) that $\lambda_{\operatorname{lin}}(k)$ is strictly decreasing in $k$ and that

$$
\lim _{k \rightarrow 0} \lambda_{\operatorname{lin}}(k)=-\frac{1}{3} \sqrt{3}>-1, \quad \lim _{k \rightarrow \infty} \lambda_{\operatorname{lin}}(k)=-\infty .
$$


Since $\lambda_{\infty}^{*}<-1$ by (4.28) there is a unique value $k_{0}$ such that (4.29) holds. By (4.29), (4.33) and (4.37) we deduce that

$$
c_{\operatorname{lin}}\left(k_{0}\right)=\bar{c}_{k_{0}}\left(\lambda_{\operatorname{lin}}\left(k_{0}\right)\right)=\bar{c}_{k_{0}}\left(\lambda_{\infty}^{*}\right)=c_{\infty},
$$

and by (4.37), (4.38) that

$$
c_{\text {lin }}\left(k_{0}\right)=\bar{c}_{k_{0}}\left(\lambda_{\infty}\left(k_{0}\right)\right) .
$$

By (4.33), (4.34) and (4.29) this yields

$$
\lambda_{\infty}\left(k_{0}\right)=\lambda_{\infty}^{*} .
$$

Finally one derives from (4.29), (4.36) and (4.38) that $k_{0}$ is given by

$$
k_{0}=\frac{1+2 \gamma}{\gamma^{2}} \text {. }
$$

To prove the inequalities (4.30), (4.32) we first observe that (4.37) implies that for $k \neq k_{0}$ there is a $\lambda$ between $\lambda_{\infty}^{*}$ and $\lambda_{\infty}(k)$ such that $\bar{c}_{k}^{\prime}(\lambda)=0$. By (4.10), (4.11) we conclude that $\lambda=\lambda_{\operatorname{lin}}(k)$ and deduce that $\lambda_{\operatorname{lin}}(k)$ lies between $\lambda_{\infty}^{*}$ and $\lambda_{\infty}(k)$. Since $\lambda_{\operatorname{lin}}(k)$ is monotonically decreasing in $k$ we obtain from (4.29) that

$$
\lambda_{\infty}\left(k_{1}\right)>\lambda_{\infty}^{*}, \quad \lambda_{\infty}\left(k_{2}\right)<\lambda_{\infty}^{*},
$$

which proves (4.30), (4.32).

The conclusions of Lemma 4.10 are illustrated in Figure 2

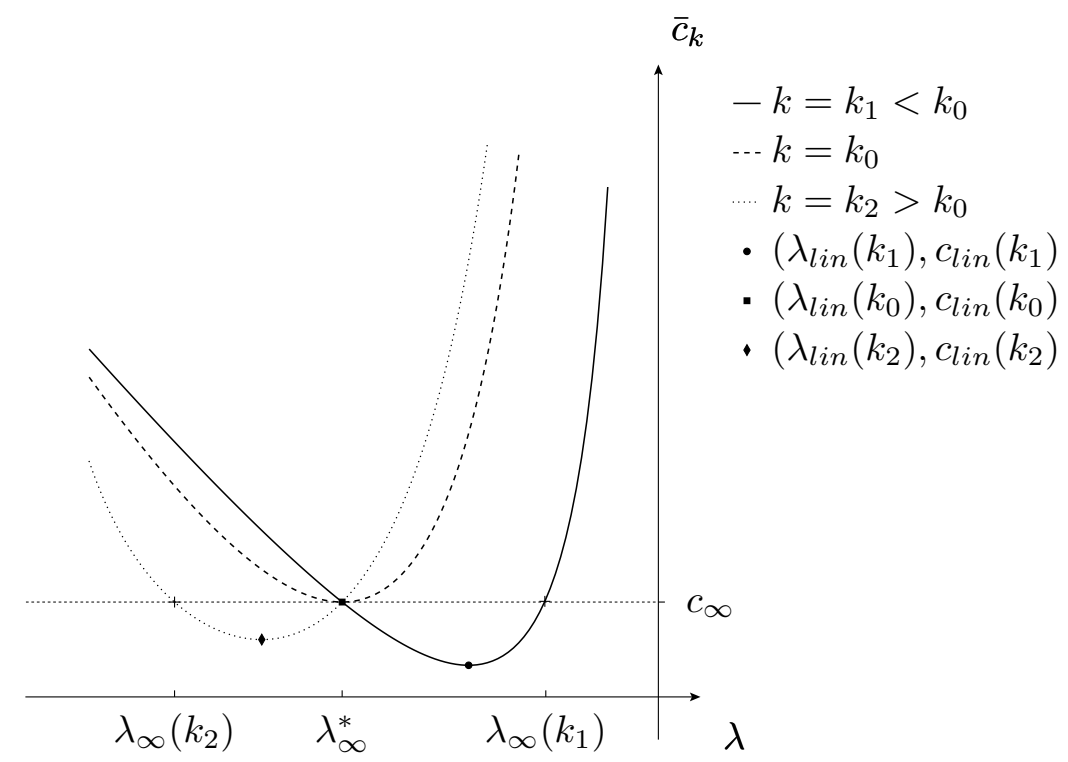

Figure 2. Graph of the functions $\bar{c}_{k}$ for different values of $k$ and the decay rates $\lambda_{\infty}(k)$ of the travelling waves with speed $c_{\infty}$ as found in Proposition 4.7 .

By the previous results we can now compare the decay of two different travelling waves as $x \rightarrow+\infty$, similarly as in Corollary 4.5 for the convergence to unity as $x \rightarrow-\infty$. 
Lemma 4.11. Let $k>k_{0}$ and assume that $(c, u, w)$ is a monotone travelling wave with speed $c<c_{\infty}$. Then, as $x \rightarrow+\infty, u(x), w(x)$ decay slower to zero than does the travelling wave $u_{0}(x), w_{0}(x)$ obtained in Proposition 4.7 .

Proof. By (4.10) the function $\bar{c}_{k}$ is monotonically decreasing for $\lambda<\lambda_{\operatorname{lin}}(k)$. From (4.32) and (4.37) we therefore deduce that

$$
\bar{c}_{k}(\lambda)>\bar{c}_{k}\left(\lambda_{\infty}(k)\right)=c_{\infty} \quad \text { for all } \lambda<\lambda_{\infty}(k) .
$$

Since we have assumed that $c<c_{\infty}$ this implies that the travelling wave $(c, u, w)$ decays with a rate $\lambda>\lambda_{\infty}(k)$. On the other hand, by Proposition 4.7, the rate of the exponential deacy of $u_{0}, w_{0}$ as $x \rightarrow+\infty$ is given by $\lambda_{\infty}(k)$.

4.4. The nonlinear selection regime. In this section we prove that for $k>k_{0}$, where $k_{0}$ is given in Lemma 4.10, the minimal speed of travelling waves for the reaction-diffusion system (1.1), (1.2) is identical to the minimal speed of travelling waves for the Stefan-like limit problem (3.2)-(3.5). In particular there is nonlinear selection of the minimal speed for $k>k_{0}$. This result follows from a comparison principle which is formulated in the next Theorem. In general, invariant region arguments do not apply for elliptic systems, but here a shift parameter is chosen to play the role of the time parameter in the proof of comparison principles for parabolic systems.

Theorem 4.12. Let $\left(c_{1}, u_{1}, w_{1}\right)$ and $\left(c_{2}, u_{2}, w_{2}\right)$ be two monotone travelling waves and assume that $c_{1}<c_{2}$. Let $\lambda_{1}, \lambda_{2}$ denote the decay rates at $+\infty$ of $u_{1}, w_{1}$ and $u_{2}, w_{2}$ respectively. Then $\lambda_{1} \leq \lambda_{2}$ holds and, as $x$ tends to infinity, $u_{1}(x), w_{1}(x)$ cannot converge exponentially slower to zero than $u_{2}(x), w_{2}(x)$ do.

In particular, a travelling wave has minimal speed if and only if its decay rate at $+\infty$ is the minimal one among all travelling waves.

Proof. Let us assume that $\lambda_{1}>\lambda_{2}$ holds. Since $c_{1}<c_{2}$ the travelling wave $\left(u_{1}, w_{1}\right)$ converges by Corollary 4.5 faster to $(1,1)$ as $x \rightarrow-\infty$ than does $\left(u_{2}, w_{2}\right)$. In particular

$$
\left(u_{1}, w_{1}\right)(x)>\left(u_{2}, w_{2}\right)(x) \quad \text { for sufficiently small } x<0 .
$$

Since we have assumed that $\lambda_{1}>\lambda_{2}$, the decay of $\left(u_{1}, w_{1}\right)$ at $+\infty$ is slower than the decay of $\left(u_{2}, w_{2}\right)$ and we deduce from Lemma 4.1 that

$$
\left(u_{1}, w_{1}\right)(x)>\left(u_{2}, w_{2}\right)(x) \quad \text { for sufficiently large } x>0 .
$$

This implies that there is a shift $x_{0}$, such that for $\left(\tilde{u}_{1}, \tilde{w}_{1}\right)=\left(u_{1}, w_{1}\right)\left(.+x_{0}\right)$

$$
\tilde{u}_{1} \geq u_{2}, \quad \tilde{w}_{1} \geq w_{2} \quad \text { on } \mathbb{R}
$$

holds and such that there exists a $x_{1} \in \mathbb{R}$ with

$$
\tilde{u}_{1}\left(x_{1}\right)=u_{2}\left(x_{1}\right) \quad \text { or } \quad \tilde{w}_{1}\left(x_{1}\right)=w_{2}\left(x_{1}\right) .
$$

From equations (2.1), (2.2) we obtain for $U:=u_{2}-\tilde{u}_{1}, W:=w_{2}-\tilde{w}_{1}$

$$
\begin{aligned}
& 0=U^{\prime \prime}+c U^{\prime}-\left(c_{1}-c_{2}\right) u_{2}^{\prime}-U+W-\gamma k\left(1-w_{2}\right) U+\gamma k \tilde{u}_{1} W, \\
& 0=c W^{\prime}-\left(c_{1}-c_{2}\right) w_{2}^{\prime}+k\left(1-w_{2}\right) U-k \tilde{u}_{1} W .
\end{aligned}
$$

Assume that $\tilde{u}_{1}\left(x_{1}\right)=u_{2}\left(x_{1}\right)$, which gives

$$
U\left(x_{1}\right)=\max _{x} U(x)=0
$$


and $U^{\prime}\left(x_{1}\right)=0, U^{\prime \prime}\left(x_{1}\right) \leq 0$. Then (4.40) yields

$$
0 \leq-\left(c_{1}-c_{2}\right) u_{2}^{\prime}\left(x_{1}\right)<0
$$

which is a contradiction.

If $\tilde{w}_{1}\left(x_{1}\right)=w_{2}\left(x_{1}\right)$ then

$$
W\left(x_{2}\right)=\max _{x} W(x)=0
$$

implies $W^{\prime}\left(x_{2}\right)=0$ and (4.41) yields

$$
0 \leq-\left(c_{1}-c_{2}\right) w_{1}^{\prime}\left(x_{2}\right)<0,
$$

which is also a contradiction. Thus we deduce that $\lambda_{1} \leq \lambda_{2}$.

The final conclusion of the Theorem follows now by a contradiction argument.

The comparison principle Theorem 4.12 and Lemma 4.11 imply that for $k>k_{0}$ no monotone travelling wave exists with lower speed than $c_{\infty}$.

Corollary 4.13. For $k \geq k_{0}$ the minimal speed of travelling waves is given by

$$
c_{\min }(k)=c_{\infty} .
$$

Proof. Assume there is a monotone travelling wave $(c, u, w)$ with $c<c_{\infty}$ and let $\left(c_{\infty}, u_{0}, w_{0}\right)$ be the monotone travelling wave with speed $c_{\infty}$ which we have found in Proposition 4.7. By Lemma 4.11 the functions $(u, w)$ decay slower to zero at $+\infty$ than $u_{\infty}, w_{\infty}$ do, which is a contradiction to Theorem 4.12. Therefore $c_{\min }(k) \geq c_{\infty}$ holds and, recalling (4.27), the conclusion follows.

\section{Travelling WAVES IN THE FAST-DEgRADATION-RATE Limit}

We complete our investigations by proving that the travelling waves of (1.1), (1.2) are, for large values of $k$, close to a travelling waves of the limit problem (3.1)-(3.5) .

Proposition 5.1. Let $\left(c, u_{k}, w_{k}\right), k \in \mathbb{N}$, be a sequence of monotone travelling waves for (1.1), (1.2) with speed $c \geq c_{\infty}$ and

$$
u_{k}(0)=\frac{1}{2} \quad \text { for all } k>0
$$

Then, as $k$ tends to infinity,

$$
\begin{aligned}
& u_{k} \rightarrow U \text { uniformly, } \\
& u_{k} \rightarrow U \quad \text { in } C_{\mathrm{loc}}^{0, \beta}(\mathbb{R}) \text {, for all } 0<\beta<1 \text {, } \\
& w_{k} \rightarrow W \quad \text { in } L_{\text {loc }}^{p}(\mathbb{R}) \text {, for all } 1 \leq p<\infty \text {, }
\end{aligned}
$$

where $U, W$ is the unique travelling-wave solution of the limit problem with speed $c$ and $U(0)=1 / 2$.

Proof. We recall that $u_{k}^{\prime}, w_{k}^{\prime}<0$, that $0<u_{k}, w_{k}<1$ and that by (2.17), (2.20)

$$
-u_{k}^{\prime}<\mu
$$


holds uniformly in $k$, where $\mu$ was defined in (2.15). This yields the existence of a subsequence $k_{i} \rightarrow \infty(i \rightarrow \infty)$ and monotone decreasing functions $u, w$ with $0 \leq u, w \leq 1$ and $u \in H_{\text {loc }}^{1, \infty}(\mathbb{R})$ such that

$$
\begin{array}{lll}
u_{k_{i}} \rightarrow u & \text { in } C_{\mathrm{loc}}^{0, \beta}(\mathbb{R}) & \text { for all } 0<\beta<1, \\
u_{k_{i}}^{\prime} \rightarrow u^{\prime} & \text { in } L_{\mathrm{loc}}^{p}(\mathbb{R}) & \text { for all } 1 \leq p<\infty, \\
u_{k_{i}} \rightarrow u & \text { uniformly in } \mathbb{R} \text { for all } 1 \leq p<\infty \\
w_{k_{i}} \rightarrow w & \text { in } L_{\mathrm{loc}}^{p}(\mathbb{R}) & \text { for all } 1 \leq p<\infty,
\end{array}
$$

Integrating equation (2.2) over $\mathbb{R}$, we obtain

$$
\int_{-\infty}^{\infty} u_{k_{i}}\left(1-w_{k_{i}}\right)=\frac{c}{k_{i}}
$$

and by Fatou's Lemma we see that

$$
\int_{-\infty}^{\infty} u(1-w) \leq \liminf _{i \rightarrow \infty} \int_{-\infty}^{\infty} u_{k_{i}}\left(1-w_{k_{i}}\right)=0
$$

which implies that

$$
u(1-w)=0 \quad \text { almost everywhere in } \mathbb{R} .
$$

By (5.3), (5.6) we obtain that $u(0)=1 / 2$ and $u(x) \geq 1 / 2$ for $x<0$. From (5.11) we deduce that

$$
w(x)=1 \quad \text { for } x<0 .
$$

The equations (2.1) and (5.5), (5.10) yield the estimate

$$
\begin{aligned}
\int_{-\infty}^{0}\left(1-u_{k_{i}}\right) & =-u_{k_{i}}^{\prime}(0)-c u_{k_{i}}(0)+c+\int_{-\infty}^{0}\left(1-w_{k_{i}}\right)+\int_{-\infty}^{0} \gamma k u_{k_{i}}\left(1-w_{k_{i}}\right) \\
& \leq C(c, \gamma)
\end{aligned}
$$

and we obtain that $(1-u) \in L^{1}((-\infty, 0))$ and $u(x) \rightarrow 1$ as $x \rightarrow-\infty$. By (2.1) and (5.5), (5.10)

$$
\int_{0}^{\infty}\left(u_{k_{i}}-w_{k_{i}}\right)=-u_{k_{i}}^{\prime}(0)-\frac{c}{2}-\gamma k_{i} \int_{0}^{\infty} u_{k_{i}}\left(1-w_{k_{i}}\right) \geq-C(\gamma, c)
$$

holds and we further deduce from (5.10) that

$$
\int_{0}^{\infty}\left(u_{k_{i}}-\frac{1}{2} w_{k_{i}}\right) \leq \int_{0}^{\infty} u_{k_{i}}\left(1-w_{k_{i}}\right) \leq \frac{c}{k_{i}}
$$

which gives, substracting (5.13),

$$
\int_{0}^{\infty} w_{k_{i}} \leq C(\gamma, c)
$$

for $k>1$. Further we find from (5.14) that

$$
\int_{0}^{\infty} u_{k_{i}} \leq C(\gamma, c)
$$

holds. By Fatou's Lemma this implies that $u, w \in L^{1}((0, \infty))$ and $u(x), w(x) \rightarrow 0$ as $x \rightarrow \infty$. By (5.6) and since the limits as $x \rightarrow \pm \infty$ of $u_{k_{i}}, U$ coincide, we deduce 
(5.2) from [Die77, Lemma 2.4].

The equations (2.1), (2.2) yield that $u_{k_{i}}, w_{k_{i}}$ satisfy

$$
0=\int_{\mathbb{R}}\left(u_{k_{i}}^{\prime}+c\left(u_{k_{i}}+\gamma w_{k_{i}}\right)\right) \eta^{\prime}+\left(u_{k_{i}}-w_{k_{i}}\right) \eta
$$

for all $\eta \in C_{c}^{\infty}(\mathbb{R})$ and, according to (5.6)-(5.9), we can pass in this equation to the limit $i \rightarrow \infty$. This yields

$$
0=\int_{\mathbb{R}}\left(u^{\prime}+c(u+\gamma w)\right) \eta^{\prime}+(u-w) \eta .
$$

Since $u, w$ are monotone decreasing from unity to zero and satisfy (5.11), we deduce that there is a $x_{0} \in \mathbb{R}$ such that

$$
\begin{array}{ll}
u(x)=0 & \text { for } x \geq x_{0}, \\
u(x)>0, w(x)=1 & \text { for } x<x_{0} .
\end{array}
$$

Therefore (5.17) yields that

$$
\begin{array}{ll}
0=u^{\prime \prime}+c u^{\prime}-u+1 & \text { for } x<x_{0}, \\
0=\gamma c w^{\prime}+w & \text { for } x>x_{0},
\end{array}
$$

and that the jump condition

$$
\gamma c\left(1-\lim _{x \downarrow x_{0}} w(x)\right)=-\lim _{x \uparrow x_{0}} u^{\prime}(x)
$$

has to be satisfied. This shows that $u, w$ is a travelling wave with speed $c$ of the limit system (3.2), (3.3).

\section{Conclusions}

We conclude our investigations with a brief summary and discussion of our results.

6.1. Slow and fast decay at the threshold $c=c_{\infty}$. Our results on the existence of travelling waves for the system (1.1), (1.2) and the selection mechanism of the minimal speed are summarized in Figure 3. As in the preceding figure we have plotted the functions $\bar{c}_{k}$ which give for a travelling wave with speed $c$ the possible rates $\lambda$ of the exponential decay to zero at $x \rightarrow+\infty$. By the circles, squares and diamonds in Figure 3 we have indicated the decay rates which in fact are realized by a travelling wave: presuming that there is linear selection for $k<k_{0}$ travelling waves exists for all speeds $c \geq c_{\text {lin. }}$. By Theorem 4.12 the decay rates of these travelling waves corresponds to values on the increasing branch of $\left(\lambda, c_{k}(\lambda)\right)$. This behaviour changes for $k>k_{0}$ : the decay rate of $u_{0}, w_{0}$ is on the decreasing branch of the solution curve. By Corollary $4.13 c_{\infty}$ is the minimal speed and the decay rates of travelling waves with larger speeds are on the increasing branch.

To give an explanation of what happens in the nonlinear selection regime $k>k_{0}$ if the speed $c$ falls below the minimal speed $c_{\min }(k)=c_{\infty}$ we consider the linearization of (1.1), (1.2) at $\left(u^{\prime}, u, w\right)=(0,0,0)$, which was given in (4.4). For all speeds $c$ in a neighbourhood of $c_{\infty}$ the linearized system has two negative eigenvalues; the stable manifold for (1.1), (1.2) at $\left(u^{\prime}, u, w\right)=(0,0,0)$ is two-dimensional. One checks that the eigenspace corresponding to a negative eigenvalue intersects with the set $\left\{z=\left(z_{1}, z_{2}, z_{3}\right): z_{1}<0, z_{2}>0, z_{3}>0\right\}$. A monotone travelling wave exists 


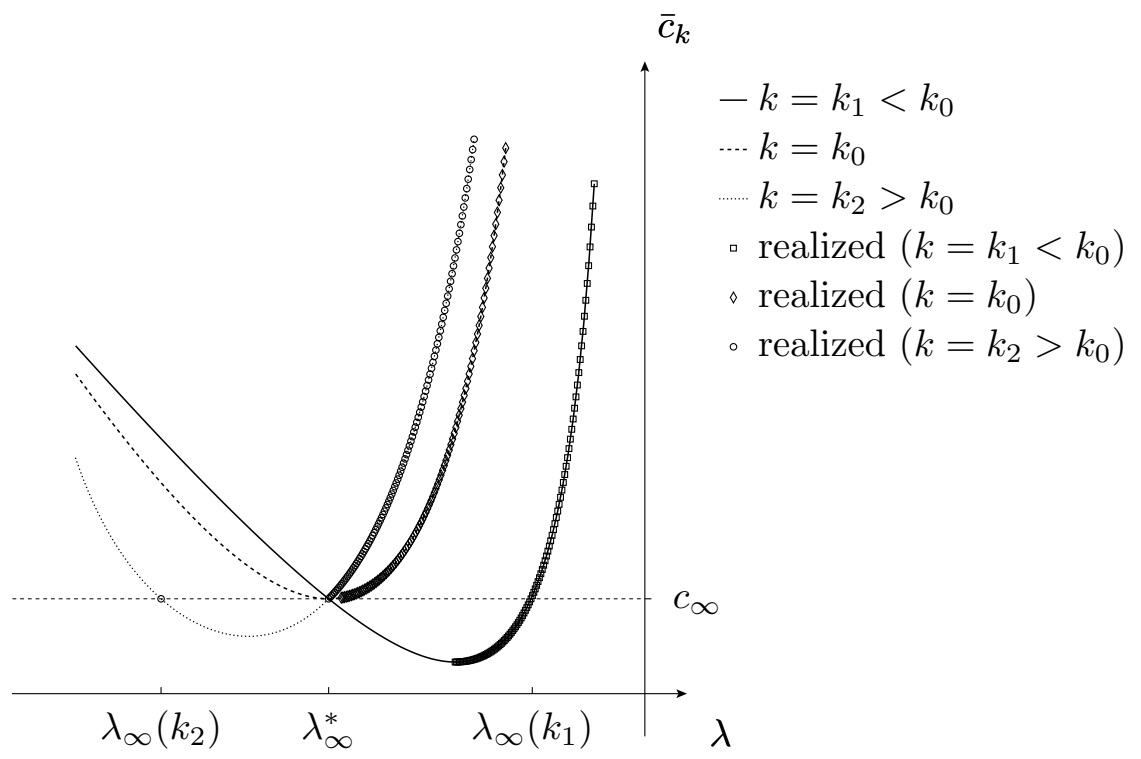

Figure 3 . Graph of the functions $\bar{c}_{k}$ for different values of $k$ and the points on the graph that are realized by a travelling wave.

for $c>c_{0}$ and approaches $(0,0,0)$ tangentially to the eigenspace corresponding to the larger negative eigenvalue ('slow decay'), see Figure 3. We expect also for $c<c_{\infty}$ an orbit $\left(u^{\prime}, u, w\right)$ connecting $(0,1,1)$ with $(0,0,0)$. Such an orbit will also converge to $(0,0,0)$ tangentially to the eigenspace corresponding to the larger negative eigenvalue but comes from the 'wrong' side, taking negative values for $u, w$. For the threshold $c=c_{\infty}$ there still exists a monotone travelling wave; this travelling wave approaches $(0,0,0)$ tangentially to the eigenspace of the smaller negative eigenvalue ('fast decay').

6.2. Discussion. Travelling waves often determine the long-time behaviour of solutions for the initial-value problem for (1.1), (1.2). Typically, for solutions with sufficiently fast decaying initial data, the propagation speed of pertubations from the unstable equilibrium is given by the minimal speed of travelling waves. The proof of such a result, as well as the uniqueness of travelling waves, for the system (1.1), (1.2) is not in the scope of the present article. Nevertheless our result that the travelling wave with minimal speed has the fastest decay supports that conjecture.

For the reaction-diffusion system (1.1), (1.2) in arbitrary space-dimension $n$ our analysis yields a family of supersolutions: Consider for $c \geq c_{\infty}$ a travelling wave $u, w$ with speed $c$ and an arbitrary real number $r \in \mathbb{R}$. The functions $\bar{u}, \bar{w}$ defined by

$$
\bar{u}(x, t):=u(|x|-c t-r), \quad \bar{w}(x, t):=w(|x|-c t-r)
$$

satisfy

$$
\begin{aligned}
\partial_{t} u-\Delta u+u-w+\gamma k u(1-w) & =-\frac{n-1}{|x|} \bar{u}^{\prime}, \\
\partial_{t} w-k u(1-w) & =0 .
\end{aligned}
$$


Since $\bar{u}^{\prime}$ is negative $(\bar{u}, \bar{w})$ is a supersolution. In order to construct a subsolution one has to control the dimension-depending correction term in (6.1).

In view of the applications, the robustness of the wave-speed to changes in the parameter values is a valuable feature: see the explicit formula (3.15) for the minimal speed and Proposition 5.1. For the mathematical analysis of reaction-diffusion systems and the selection of the minimal speed the model that we have derived is a good paradigm. We prove that nonlinear selection occurs and determine explicitly the minimal speed. One crucial ingredient is the exact first integral obtained in Lemma 4.6. which is a special property of the system (1.1), (1.2). Other results, in particular the comparison principle Theorem 4.12 and the observation that the fastest decay is realized by a travelling wave with minimal speed, can be extended to general monotone systems.

\section{REFERENCES}

[AW75] D. G. Aronson and H. F. Weinberger. Nonlinear diffusion in population genetics, combustion, and nerve pulse propagation. In Partial differential equations and related topics (Program, Tulane Univ., New Orleans, La., 1974), pages 5-49. Lecture Notes in Math., Vol. 446. Springer, Berlin, 1975.

[BD94] R. D. Benguria and M. C. Depassier. Validity of the linear speed selection mechanism for fronts of the nonlinear diffusion equation. Phys. Rev. Lett., 73:2272-2274, 1994.

[CL55] E. A. Coddington and N. Levinson. Theory of ordinary differential equations. McGraw-Hill Book Company, New York-Toronto-London, 1955.

[Die77] O. Diekmann. Limiting behaviour in an epidemic model. Nonlinear Anal., 1:459-470, 1976/77.

[EvS00] U. Ebert and W. van Saarloos. Front propagation into unstable states: universal algebraic convergence towards uniformly translating pulled fronts. Phys. D, 146:1-99, 2000.

[Fis37] R. A. Fisher. The wave of advance of advantageous genes. Ann. Eugenics, 7:355-369, 1937.

[HKR07] D. Hilhorst, J. R. King, and M. Röger. Mathematical analysis of a model describing the invasion of bacteria in burn wounds. Nonlinear Anal. (TMA), 66:1118-1140, 2007.

[HR75] K. P. Hadeler and F. Rothe. Travelling fronts in nonlinear diffusion equations. $J$. Math. Biol., 2:251-263, 1975.

$\left[\mathrm{KKC}^{+} 03\right]$ J. R. King, A. J. Koerber, J. M. Croft, J. P. Ward, R. E. Sockett, and P. Williams. Modelling host tissue degradation by extracellular bacterial pathogens. Math. Med. Biol., 20:227-260, 2003.

[KPP37] A. N. Kolmogorov, I. G. Petrovsky, and N. S. Piskunov. Etude de l'eq́uation de la diffusion avec croissance de la quantité de matière et son application à un problème biologique. Moskow Univ. Math. Bull., 1:1-25, 1937.

[LMN04] M. Lucia, C. B. Muratov, and M. Novaga. Linear vs. nonlinear selection for the propagation speed of the solutions of scalar reaction-diffusion equations invading an unstable equilibrium. Comm. Pure Appl. Math., 57:616-636, 2004.

[Mur04] C. B. Muratov. A global variational structure and propagation of disturbances in reaction-diffusion systems of gradient type. Discrete Contin. Dyn. Syst. Ser. B, 4:867892, 2004.

[Per01] L. Perko. Differential equations and dynamical systems, volume 7 of Texts in Applied Mathematics. Springer-Verlag, New York, 2001.

[Roq97] J.-M. Roquejoffre. Eventual monotonicity and convergence to travelling fronts for the solutions of parabolic equations in cylinders. Ann. Inst. H. Poincaré Anal. Non Linéaire, 14:499-552, 1997.

[Rot81] F. Rothe. Convergence to pushed fronts. Rocky Mountain J. Math., 11:617-633, 1981.

[Sto76] A. N. Stokes. On two types of moving front in quasilinear diffusion. Math. Biosci., 31:307-315, 1976.

[vS89] W. van Saarloos. Front propagation into unstable states. II. Linear versus nonlinear marginal stability and rate of convergence. Phys. Rev. A, 39:6367-6390, 1989. 
[vS03] W. van Saarloos. Front propagation into unstable states. Physics Reports, 386:29-222, 2003.

[VVV94] A. I. Volpert, V. A. Volpert, and V. A. Volpert. Traveling wave solutions of parabolic systems, volume 140 of Translations of Mathematical Monographs. American Mathematical Society, Providence, RI, 1994.

$\left[W_{K K}^{+} 04\right]$ J. P. Ward, J. R. King, A. J. Koerber, J. M. Croft, R. E. Sockett, and P. Williams. Cell-signalling repression in bacterial quorum sensing. Math. Med. Biol., 21:169-204, 2004 .

Danielle Hilhorst, CNRS and laboratoire de Mathématiques, Université de ParisSud, 91405 Orsay Cedex, France

John R. King, Centre for Mathematical Medicine, Theoretical Mechanics Section, School of Mathematical Sciences, University of Nottingham, Nottingham, NG7 2RD, UK

Matthias Röger, Max Planck Institute for Mathematics in the Sciences, Inselstr. 22, D-04103 LEIPZIG 